\title{
Subjective sleep disorders and daytime sleepiness in patients with restrictive type anorexia nervosa and effects on quality of life: a case-control study.
}

Andrea Romigi ( $\square$ andrea.romigi@gmail.com )

IRCCS Neuromed https://orcid.org/0000-0002-4505-2600

Nicola B. Mercuri

University of Rome Tor Vergata: Universita degli Studi di Roma Tor Vergata

Marco Caccamo

IRCCS Neurological Institute of Southern Italy NEUROMED: NEUROMED

\section{Federica Testa}

IRCCS Neurological Institute of Southern Italy NEUROMED: NEUROMED

\section{Giuseppe Vitrani}

IRCCS Neurological Institute of Southern Italy NEUROMED: NEUROMED

\section{Maria Carmela Tripaldi}

University of Rome Tor Vergata: Universita degli Studi di Roma Tor Vergata

\section{Diego Centonze}

IRCCS Neurological Institute of Southern Italy NEUROMED: NEUROMED

\section{Fabrizio Jacoangeli}

University of Rome Tor Vergata: Universita degli Studi di Roma Tor Vergata

\section{Original Article}

Keywords: anorexia nervosa, restrictive type, Sleep, sleepiness, quality of life, SF36

Posted Date: February 23rd, 2021

DOI: https://doi.org/10.21203/rs.3.rs-167173/v1

License: (1) (1) This work is licensed under a Creative Commons Attribution 4.0 International License.

Read Full License

Version of Record: A version of this preprint was published at Sleep and Biological Rhythms on November 9th, 2021. See the published version at https://doi.org/10.1007/s41105-021-00352-7. 


\section{Abstract}

Purpose To evaluate sleep disorders and daytime drowsiness in a cohort of patients affected by anorexia nervosa (AN) and their impact on health-related quality of life (HRQoL).

Method We evaluated AN patients and healthy controls (C) by the Pittsburgh Sleep Quality Index (PSQI), Epworth Sleepiness Scale, Beck Depression Index. We also utilized the Short-Form Health Survey (SF-36) questionnaire to assess HRQoL in both AN and C.

Results: Twenty-eight out of 34 AN patients (82.3\%) had a pathological PSQI score while 10/34 (29.4\%) healthy subjects $(p<0.0001)$. The overall PSQI score was significantly higher in AN than in the controls $(p=0.0004)$. The components sleep quality $(p=0.008)$, sleep duration $(p=0.03)$, sleep efficiency $(p=0.004)$, sleep disturbances $(p=0.01)$ and daytime dysfunction $(p=0.002)$ were higher than C. SF36 showed significantly reduced scores of standardized physical components $(p=0.01)$ and standardized mental components $(p<0.0001)$ in AN, while physical function $(p=0.0001)$, physical role $(p=0.0005)$ and general health $(p<0.0001)$, vitality $(p=0.001)$, social functioning $(p=0.0006)$ emotional role $(p=0.002)$ and mental health $(p<0.0001)$ were significantly decreased in AN. We found a significant correlation between the PSQI score and the physical role $(r=-0.35, p=0.03)$ and education $(r=0.38, p=0.02)$.

Conclusion We demonstrated low sleep quality lacking subjective daytime sleepiness in AN. Sleep quality correlated significantly with HRQoL (physical role) and level of education.

Level of evidence: level III case-control study

\section{Introduction}

Anorexia nervosa (AN) is a severe psychiatric disorder characterized by refusal to maintain proper body weight, an intense fear of gaining weight despite the patients are underweight due to a distorted perception of body image. AN generally emerges during adolescence and adult age, and it represents the most common psychiatric disorder in young girls aged 15-19 years [1,2]. AN include two different subtypes; the restricting-type (AN-R) and the Binge-Eating/Purging Type. In AN-R, a reduction in food intake without binge-eating or purging behaviour accomplishes weight loss $[2,3]$.

Sleep disorders are frequently associated with psychiatric diseases [4]. The early recognition of sleep complaints and their management may improve the outcome of the disease itself and prevent psychiatric illness relapse[4-7]. AN is often associated with sleep impairment, despite a few studies on the prevalence of sleep disturbances in AN [7]. Some authors described sleep problems as independent clinical syndromes in AN[8]. Hypothalamic nuclei and neuropeptides (such as hypocretin) modulate the sleep propensity, the sleep-wake cycle, feeding, and endocrinal system. Hypothalamic dysregulation might impair the sleep and eating homeostasis by anorexigenic and orexigenic hormones [7,9-13]. 
However, previous studies regarding sleep and AN showed conflicting results[ 7,14$]$. Polysomnographic studies showed inconsistent results. The main findings were a reduction of both slow-wave sleep and REM sleep and shorter REM sleep latency [7,14-18], sleep microstructure impairment [19] or no significant changes in patients with AN and controls [20-22]. Previous studies failed to clarify the pathogenesis of sleep disorders in AN patients. Alteration of neurotransmitters (i.e. serotonin, norepinephrine, dopamine, opioids and endocannabinoids, and the close correlation between the midbrain and hypothalamic areas may be involved $[9,10,23]$.

The goal of our study was to evaluate sleep disturbances and daytime sleepiness by subjective scales in a homogeneous sample of patients with AN-R to assess its impact on mood and quality of life compared with a homogeneous group of healthy women matched for age, sex and education level.

\section{Methods}

\section{Subjects}

Our case-control study involved a cohort of female patients affected by AN-R and age, sex, and education matched healthy controls. The participants were female outpatients, sought treatment for their AN-R at the Clinical Nutrition and Eating Disorders Unit of University General Hospital Tor Vergata, between 1st June 2012 and $30^{\text {th }}$ September 2013. All subjects were affected by restricting subtype of AN at the time of the evaluation under the Diagnostic and Statistical Manual of Mental Disorder, $4^{\text {th }}$ Edition (DSM-IV-TR) criteria. We recruited healthy female controls matched for age, sex and education level among the high school students and university students with no medical and psychiatric or medical. Exclusion criteria for both groups were: occurrence of comorbid physical illness or any neurological disorders, psychiatric disorders other than AN-R and comorbid disorders for the patient group, and intake of drugs influencing sleep and sleepiness. All patients and controls gave written informed consent; the local Ethical Committee approved the study.

\section{Subjective scales}

All subjects (patients and controls) underwent a subjective evaluation of the overall sleep quality (Pittsburgh Sleep Quality Index, PSQI) [24-26], sleepiness (Epworth Sleepiness Scale)[27,28], quality of life Italian version of the Short-Form Health Survey (SF-36) questionnaire (4-week version) (SF36) [29,30] and depressive symptoms (Beck Depression Inventory, BDI-II)[31,32].

The PSQI is a self-rated questionnaire that assesses sleep quality and disturbance over one month. The PSQI is a 24-item scale that measures sleep disturbances according to 7 dimensions: subjective sleep quality (C1), sleep latency (C2), sleep duration (C3), habitual sleep efficiency (C4), sleep disturbances (C5), use of sleep medication (C6), and daytime dysfunction (C7). The sum of these seven areas yields the global score. The questionnaire is based on the majority of days and nights of the previous month. $A$ global score of $>5$ was an indicator of relevant sleep disturbances $[25,26]$. 
We utilized the Italian version of ESS; an eight items scale investigating daytime sleep propensity in different real-life situations, referring to the previous month; each subject filled in the scale in the morning. Each item score is from zero to three with a final scoring from 0 to 24 , and an ESS score $>10$ revealed excessive daytime sleepiness $[27,28]$.

We evaluated HRQoL by the Italian version of the SF-36 questionnaire (4-week version)[29,30]. The SF-36 is grouped in 8 multi-item scales: physical functioning (PF), role limitations due to physical problems $(\mathrm{RP})$, bodily pain (BP), general health perceptions $(\mathrm{GH})$, vitality $(\mathrm{VT})$, social functioning (SF), role limitations due to emotional problems (RE) and mental health (MH). The score of SF-36 ranged from 0 to 100,0 indicating extreme problems and 100 indicating no problems. Scores range from 0 to 100 with higher values indicating better QoL. We obtained two global components Physical Component Summary (PCS) and Mental Component Summary (MCS) by the collapse of the eight SF-36 domains [30,33].

The Beck Depression Inventory-II (BDI) is a validated self-administered scale consisting of 21 multiplechoice items. Each item is rated from zero (no symptoms) to three (most severe). The scores range from 0 to 63 . Scores are evaluated as follows: 0 to 9, the absence of depression, 10-18, mild to moderate, 1929 , moderate to severe, $30-63$, severe[31,32].

The data that support the findings of this study are available on request from the corresponding author. The data are not publicly available due to privacy or ethical restrictions.

\section{Statistical analysis}

We conducted the statistical analysis on two groups, patients with AN-R and C. We compared the demographic and clinical data of both groups (age, weight, height, BMI, and years of education) and psychometric scales BDI, PSQI, ESS, SF-36. We showed mean and standard deviation of demographic variables, medians and interquartile ranges for psychometric variables (BDI, PSQI, ESS, SF-36).

We used nonparametric unpaired Mann-Whitney and chi-square test when required to compare demographic and psychometric variables in AN and C. The patients were subdivided by the PSQI cut-off $(>5)$ in AN with sleep disorders (ANSD) and AN without sleep disorder (ANwSD). We compared the subdomains of PSQI, the domains of the SF36 and the ESS score in these two groups (ANSD and ANwSD) by nonparametric unpaired Mann-Whitney test. The nonparametric correlation Spearman test was applied to test the relationship between PSQI score and education level, SF36 and BDI. Statistical analysis was performed using the Jamovi software[34,35]. The level of statistical significance was set to values of $p<0.05$. We also reported the values of effect size $(r)$.

\section{Results}

\section{Demographic characteristics}

We recruited a total of 34 women affected by AN-R (mean age 23.3, SD 6:53 range 14-39). Mean BMI was 16.4 (SD 1.47 range 12.6 to 18.7). The control group consisted of 34 women matched for age (mean age 
23.3 SD 6.53 range 14-39), sex and education to the AN group. In particular, the group of patients (AN) showed a significantly lower BMI compared with controls $(p<0.0001)$, whereas age, sex and education were similar as required by the study design. Demographic data are summarized in Table 1.

\section{Psychometric Variables}

All patients with AN-R $(n=34)$ and all healthy subjects $(C)$ have been evaluated by psychometric scales described above. BDI-II presented significantly more severe scores in patients (AN) than healthy controls $(p=0.0001)$. The data are summarized in Table 1.

\section{Nocturnal sleep quality (PSQI)}

Patients with AN-R experienced a significant presence of sleep disorders. Of the 34 subjects with AN $28 / 34(82.3 \%)$ had a pathological PSQI score $(>5)$ while $10 / 34(29.4 \%)$ healthy subjects $(X 2=17.2, p<$ $0.0001)$.

The overall PSQI score was significantly higher in the affected group (AN-R) than in the controls $(p=$ $0.0004)$. As for the individual components, the components $C 1$ sleep quality $(p=0.008), C 3$ sleep duration $(p=0.03)$, C4 sleep efficiency $(p=0.004)$, sleep disturbances C5 $(0.01)$ and daytime dysfunction C7 ( $p=0.002)$ were higher in AN than Controls. The data are presented in Table 2. Patients with sleep complaints (PSQI > 5, ANSD $n=28$ ) and lacking sleep complaints (PSQI $\leqq 5, A N w S D n=6)$ showed no significant differences in ESS score $(p=0.96)$ and individual components of SF-36 and of the physical ( $p$ $=0.27)$ and mental $(p=0.94)$ standardized components. The values are shown in Table 3.

\section{Daytime Sleepiness (Epworth Sleepiness Scale ESS)}

Patients affected (AN-R) did not experience significant daytime drowsiness as assessed by ESS. Of the 34 subjects with AN 8/34 (23\%) had a general score of pathological ESS (> 10) while $3 / 34(8 \%)$ healthy subjects $(x 2=2.7, p=0.09)$. The total ESS score was not statistically different in the two groups (AN vs $C$, $p=0.7)$ (see Table 2).

\section{Quality of life (SF-36)}

AN-R patients presented significantly reduced scores of PCS $(p=0.01)$ and MCS $(p<0.0001)$. Considering the single components, $\operatorname{PF}(p=0.0001), \operatorname{RP}(p=0.0005)$ and $\mathrm{GH}(p<0.0001)$ were significantly lower than the controls. VT $(p=0.001), S F(p=0.0006), \operatorname{RE}(p=0.002)$ and MH $(p<0.0001)$ were significantly reduced in AN-R. The data are presented in Table 2.

\section{Comparison between ANSD and ANwSD}

No significant differences were found between sleep complaints (ANSD) and not sleep complaints (ANwSD) regarding ESS, BDI-II and SF36 (see table 3). 
The correlations between the PSQI and SF36, BMI, education and BDI score, are shown in Table 4. A significant correlation between the PSQI score and the RP was found $(r=-0.35, p=0.03$ see table 3 and fig.1a). A significant correlation between years of education and PSQI $(r=0.38, p=0.02$ Table 4 and fig.1b) was also found.

\section{Discussion}

Sleep research in AN addressed two crucial issues: (1) the effects of forced fasting in the AN and rapid food fluctuations in bulimia nervosa on the regulatory processes of the sleep-wake cycle and (2) the search for a significant relationship between the neurobiology of AN and major depression. At present, the second question seems to have found some answers, since most of the available evidence underlines the idea that $\mathrm{AN}$ and affective disorders are two distinct entities. On the other hand, as regards the effects of forced fasting on sleep regulation, recent research on animal models and humans seems to show that the AN produces substantial sleep fragmentation and deep sleep reduction [14]. Although several peptides appear to be involved in these regulatory processes (e.g., CCK, orexin, leptin), their mechanism of action is still unclear. In our controlled study by validated subjective scales, we confirm a significant tendency to low sleep quality as shown by the significantly higher overall PSQI score in the patient group (AN-R) than controls. Sleep disorders are significantly increased, affecting approximately $83 \%$ of AN-R compared to $29.4 \%$ of the healthy subjects. Although this observation is expected (Leuer and Krieg 2004), PSQI seems to be a useful tool documenting the presence of disturbed sleep in the AN-R. It is well known that patients with AN-R rarely report sleep disorders as part of the clinical picture. It is probably due to AN$\mathrm{R}$ patients' common belief that a shortened sleep period and time in bed may allow being longer active and, therefore, increase caloric expenditure. On the other hand, patients affected by bulimia nervosa may delay sleep onset time of one hour or more because of compulsive eating episodes in the late evening or early morning $[7,14,17,21]$. These different sleep profiles could account for conflicting results obtained in several studies conducted in heterogeneous patient groups that included forms of "primary" or "secondary" AN to other psychiatric or organic disorders or including patients with bulimia nervosa or nonrestrictive AN $[14,17,36]$. The paucity of studies conducted in patients with restrictive AN confirmed the sleep fragmentation (mainly due to early awakening) and a reduction of slow-wave sleep (SWS) and REM sleep, higher REM sleep latency [14,15](Foster et al., 1976; Nobili et al., 1999; Levy et al. 1987; Lauer and Krieg, 2004). Reduction of slow-wave activity (a quantitative measure of deep sleep) correlated with weight and BMI $[7,14,16]$. Regarding PSQI, we found a significant increase in the overall sleep quality component (C1), sleep duration (C3), sleep efficiency (C4) and sleep disorders (C5) in our AN-R sample. These components are closely related to the AN-related sleep profile, where AN-R patients would gain active time and, reduce total sleep time, time in bed to increase caloric expenditure. The consequences of prolonged sleep deprivation and poor sleep quality may induce daytime dysfunction as highlighted by the significantly higher C7 component (daytime dysfunction) of PSQI. Diurnal sleepiness is not significantly affected in AN-R compared to controls as shown by the Epworth Sleepiness Scale. Animal models confirmed that the induction of 'satiety' (i.e. intake of food, CCK applications, bombesin and leptin) prompts an increase in EDS and deep sleep (short sleep onset latency, an increase of SWS, decrease in 
REM sleep). On the other hand prolonged food deprivation and the application of orexin (induction of 'hunger') result in an increased wake time and light sleep, in particular, a decrease in slow-wave sleep[14,37]. Therefore, prolonged fasting in AN-R may increase vigilance confirming the lack of EDS as we showed in our AN sample. Concerning QoL, we found a significant and widespread reduction in quality of life measures in all five mental dimensions (vitality, general health, social functioning, role emotional and mental health) and 4 out of 5 physical components (physical functioning, physical role and vitality and general health) and in both Physical and Mental components summaries in AN-R compared to C. The SF-36 represents a widely validated and accepted quality of life assessment scale in different clinical areas $[30,33,38]$. AN is a chronic and often severe disorder, which significantly impact on different areas of life. AN may impair mental, physical, behavioural and socio-professional abilities. Therefore, the quality of life in AN patients is often worse than the healthy population. Quality of life can be a key factor in measuring the severity of the disease and the effectiveness of the treatment carried out. QoL's mental health components are more often affected than physical ones in AN [38]. AN-R patients may perceive fewer limitations than subjects with other eating disorders. This finding is consistent with previous reports of better SF-36 emotional well-being in those with restrictive eating behaviours. However, this result should be interpreted with caution, as it may be due to intrinsic limitations of the instruments used and/or an "artefact" produced by the psychopathological condition itself, obesity and other correlated condition rather than by a real better condition of AN $[39,40]$. We divided AN-R into two groups with (ANSD, $N=28$ ) or without sleep impairment (ANwSD, $n=6$ ) by PSQI cut-off. No significant differences were found concerning daytime drowsiness (ESS), the severity of depression (BDI-II), and quality of life (SF-36) even if the small sample limits this analysis. Lastly, we found an interesting correlation between the severity of sleep disturbances (PSQI global score) score and a physical domain of the SF-36 (physical role) and education. We stated that the SF36 physical components seemed to be less affected in the AN-R or perhaps less easily assessable either because of intrinsic limitations of the quality of life measurement instruments and the specific aspects of the psychopathology. We did not find studies correlating QoL and sleep quality in AN. In our sample, we found a negative correlation between physical role and PSQI scores, demonstrating that poor sleep quality may directly impact the physical component of the quality of life. The physical role may quantify work problems and other daily activities due to physical health $[30,33]$. Sleep impairment due to sleep fragmentation and sleep loss could negatively affect the quality of life and diurnal function, as shown by PSQI scores. Regarding the significant positive correlation between education level and severity of PSQI, previous studies report significant interactions between the social environment and the development of eating disorders. However, the results are mixed, and the social context's role in the aetiology of AN is unclear[41]. Some authors found higher parental education related to eating disorders severity $[41,42]$. However, these findings may represent a selection bias, linked to increased attention to AN in this type of family context $[41,42]$. On the contrary, sleep fragmentation and short duration of total sleep time are generally associated with lower social status and lower education years (Stamatakis and coll., 2007). •

\section{Conclusions}


Although a small sample limits this study, it represents an attempt to evaluate the relationship between sleep disorders and AN in a sample of homogenous patients with AN-R. We found significant impairment of sleep and daytime function without EDS. Both the physical and mental components of QoL are profoundly affected in AN. Sleep impairment correlated significantly with a worse quality of physical role (negative correlation) and a higher education level (positive correlation). Future studies should evaluate sleep disorders in larger AN samples, by subjective and objective evaluation (i.e. actigraphic and/or polysomnographic methods).

\section{Declarations}

\section{Acknowledgements}

We acknowledge the efforts of participants and volunteers for their support.

\section{Conflict of interest}

The authors declare that they have no conflict of interest.

\section{Research involving human participants and/or animals}

All procedures performed in studies involving human participants were in accordance with the ethical standards of the institutional and/or national research committee and with the 1964 Helsinki declaration and its later amendments or comparable ethical standards.

\section{Informed consent}

Informed consent was obtained from all individual participants included in the study

\section{References}

[1] Smink FRE, van Hoeken D, Hoek HW. Epidemiology of eating disorders: incidence, prevalence and mortality rates. Curr Psychiatry Rep 2012;14:406-14. doi:10.1007/s11920-012-0282-y.

[2] Hoek HW. Incidence, prevalence and mortality of anorexia nervosa and other eating disorders. Curr Opin Psychiatry 2006;19:389-94. doi:10.1097/01.yco.0000228759.95237.78.

[3] Van Autreve S, De Baene W, Baeken C, van Heeringen C, Vervaet M. Do restrictive and bingeing/purging subtypes of anorexia nervosa differ on central coherence and set shifting? Eur Eat Disord Rev 2013;21:308-14. doi:10.1002/erv.2233.

[4] Sateia MJ. Update on sleep and psychiatric disorders. Chest 2009;135:1370-9. doi:10.1378/chest.081834. 
[5] Ivanenko A, Johnson K. Sleep disturbances in children with psychiatric disorders. Semin Pediatr Neurol 2008;15:70-8. doi:10.1016/j.spen.2008.03.008.

[6] Abad VC, Guilleminault C. Sleep and psychiatry. Dialogues Clin Neurosci 2005;7:291-303.

[7] Padez-Vieira F, Afonso P. Sleep disturbances in anorexia nervosa. Advances in Eating Disorders 2016;4:176-88. doi:10.1080/21662630.2016.1175958.

[8] Birketvedt GS, Florholmen J, Sundsfjord J, Osterud B, Dinges D, Bilker W, et al. Behavioral and neuroendocrine characteristics of the night-eating syndrome. JAMA 1999;282:657-63.

[9] Salin-Pascual R, Gerashchenko D, Greco M, Blanco-Centurion C, Shiromani PJ. Hypothalamic regulation of sleep. Neuropsychopharmacology 2001;25:S21-7. doi:10.1016/S0893-133X(01)00318-9.

[10] Pizza F, Magnani M, Indrio C, Plazzi G. The hypocretin system and psychiatric disorders. Curr Psychiatry Rep 2014;16:433. doi:10.1007/s11920-013-0433-9.

[11] McSherry J, Ashman G. Bulimia and sleep disturbance. J Fam Pract 1990;30:102-3.

[12] Romigi A, Bari M, Liguori C, Izzi F, Rapino C, Nuccetelli M, et al. CSF levels of the endocannabinoid anandamide are reduced in patients with untreated narcolepsy type 1: a pilot study. CNS Neurol Disord Drug Targets 2020. doi:10.2174/1871527319666200309115602.

[13] Romigi A, Centonze D. Oral L-carnitine and pregnancy: real opportunity for narcoleptic women? Sleep Sci 2019;12:124. doi:10.5935/1984-0063.20190060.

[14] Lauer CJ, Krieg J-C. Sleep in eating disorders. Sleep Med Rev 2004;8:109-18. doi:10.1016/S10870792(02)00122-3.

[15] Nobili L, Baglietto MG, Beelke M, De Carli F, Di Comite R, Fiocchi I, et al. Impairment of the production of delta sleep in anorectic adolescents. Sleep 2004;27:1553-9.

[16] Pieters G, Theys P, Vandereycken W, Leroy B, Peuskens J. Sleep variables in anorexia nervosa: evolution with weight restoration. Int J Eat Disord 2004;35:342-7. doi:10.1002/eat.10256.

[17] Asaad Abdou T, Esawy HI, Abdel Razek Mohamed G, Hussein Ahmed H, Elhabiby MM, Khalil SA, et al. Sleep profile in anorexia and bulimia nervosa female patients. Sleep Med 2018;48:113-6. doi:10.1016/j.sleep.2018.03.032.

[18] Katz JL, Kuperberg A, Pollack CP, Walsh BT, Zumoff B, Weiner H. Is there a relationship between eating disorder and affective disorder? New evidence from sleep recordings. Am J Psychiatry 1984;141:753-9. doi:10.1176/ajp.141.6.753.

[19] Della Marca G, Farina B, Mennuni GF, Mazza S, Di Giannantonio M, Spadini V, et al. Microstructure of sleep in eating disorders: preliminary results. Eat Weight Disord 2004;9:77-80. doi:10.1007/bf03325049. 
[20] Latzer Y, Tzischinsky O, Epstein R. Sleep-wake monitoring in women suffering from anorexia nervosa. Eat Disord 2001;9:159-66. doi:10.1080/10640260127713.

[21] Lauer CJ, Krieg JC, Riemann D, Zulley J, Berger M. A polysomnographic study in young psychiatric inpatients: major depression, anorexia nervosa, bulimia nervosa. J Affect Disord 1990;18:235-45.

[22] Levy AB, Dixon KN, Schmidt H. Sleep architecture in anorexia nervosa and bulimia. Biol Psychiatry 1988;23:99-101.

[23] Miljic D, Pekic S, Djurovic M, Doknic M, Milic N, Casanueva FF, et al. Ghrelin has partial or no effect on appetite, growth hormone, prolactin, and cortisol release in patients with anorexia nervosa. J Clin Endocrinol Metab 2006;91:1491-5. doi:10.1210/jc.2005-2304.

[24] Romigi A, Albanese M, Placidi F, Izzi F, Liguori C, Marciani MG, et al. Sleep disorders in myotonic dystrophy type 2: a controlled polysomnographic study and self-reported questionnaires. Eur J Neurol 2014;21:929-34. doi:10.1111/ene.12226.

[25] Curcio G, Tempesta D, Scarlata S, Marzano C, Moroni F, Rossini PM, et al. Validity of the Italian version of the Pittsburgh Sleep Quality Index (PSQI). Neurol Sci 2013;34:511-9. doi:10.1007/s10072-0121085-y.

[26] Buysse DJ, Reynolds CF, Monk TH, Berman SR, Kupfer DJ. The Pittsburgh Sleep Quality Index: a new instrument for psychiatric practice and research. Psychiatry Res 1989;28:193-213. doi:10.1016/01651781(89)90047-4.

[27] Vignatelli L, Plazzi G, Barbato A, Ferini-Strambi L, Manni R, Pompei F, et al. Italian version of the Epworth sleepiness scale: external validity. Neurol Sci 2003;23:295-300. doi:10.1007/s100720300004.

[28] Johns MW. A new method for measuring daytime sleepiness: the Epworth sleepiness scale. Sleep 1991;14:540-5. doi:10.1093/sleep/14.6.540.

[29] Ware JE, Sherbourne CD. The MOS 36-item short-form health survey (SF-36). I. Conceptual framework and item selection. Med Care 1992;30:473-83.

[30] Apolone G, Mosconi P. The Italian SF-36 Health Survey: translation, validation and norming. J Clin Epidemiol 1998;51:1025-36.

[31] Goretti B, Portaccio E, Zipoli V, Hakiki B, Siracusa G, Sorbi S, et al. Coping strategies, psychological variables and their relationship with quality of life in multiple sclerosis. Neurol Sci 2009;30:15-20. doi:10.1007/s10072-008-0009-3.

[32] Beck AT, Steer RA, Ball R, Ranieri W. Comparison of Beck Depression Inventories -IA and -II in psychiatric outpatients. J Pers Assess 1996;67:588-97. doi:10.1207/s15327752jpa6703_13. 
[33] McHorney CA, Ware JE, Raczek AE. The MOS 36-Item Short-Form Health Survey (SF-36): II.

Psychometric and clinical tests of validity in measuring physical and mental health constructs. Med Care 1993;31:247-63.

[34] Sahin MD, Aybek EC. Jamovi: An Easy to Use Statistical Software for the Social Scientists. International Journal of Assessment Tools in Education 2019.

[35] The jamovi project (2020). jamovi. (Version 1.2) [Computer Software]. Retrieved from https://www.jamovi.org. Sydney, Australia: Jamovi; 2020.

[36] Kim KR, Jung Y-C, Shin M-Y, Namkoong K, Kim J-K, Lee J-H. Sleep disturbance in women with eating disorder: prevalence and clinical characteristics. Psychiatry Res 2010;176:88-90.

doi:10.1016/j.psychres.2009.03.021.

[37] Dewasmes G, Cohen-Adad F, Koubi H, Le Maho Y. Sleep changes in long-term fasting geese in relation to lipid and protein metabolism. Am J Physiol 1984;247:R663-71.

doi:10.1152/ajpregu.1984.247.4.R663.

[38] Doll HA, Petersen SE, Stewart-Brown SL. Eating disorders and emotional and physical well-being: associations between student self-reports of eating disorders and quality of life as measured by the SF36. Qual Life Res 2005;14:705-17. doi:10.1007/s11136-004-0792-0.

[39] Ágh T, Kovács G, Supina D, Pawaskar M, Herman BK, Vokó Z, et al. A systematic review of the healthrelated quality of life and economic burdens of anorexia nervosa, bulimia nervosa, and binge eating disorder. Eat Weight Disord 2016;21:353-64. doi:10.1007/s40519-016-0264-x.

[40] Sy R, Ponton K, De Marco P, Pi S, Ishak WW. Quality of life in anorexia nervosa: a review of the literature. Eat Disord 2013;21:206-22. doi:10.1080/10640266.2013.779176.

[41] Vanderlinden J, Schoevaerts K, Simons A, Van Den Eede U, Bruffaerts R, Serra R, et al. Sociodemographic and clinical characteristics of eating disorder patients treated in the specialized residential settings in Belgium. Eat Weight Disord 2020. doi:10.1007/s40519-020-00867-x.

[42] Ahrén JC, Chiesa F, Af Klinteberg B, Koupil I. Psychosocial determinants and family background in anorexia nervosa--results from the Stockholm Birth Cohort Study. Int J Eat Disord 2012;45:362-9. doi:10.1002/eat.20953.

\section{Tables}

Table 1 Demographic characteristics of AN and healthy controls 


\begin{tabular}{|llll|}
\hline & AN-R $(\mathrm{N}=34)$ & \multicolumn{1}{c}{$\mathbf{C ~ ( n = 3 4 )}$} & P \\
\hline Age & $23.3 \pm 6.53$ & $23.3 \pm 6.53$ & NS \\
\hline Gender & $\mathbf{3 4 ~ F}$ & $\mathbf{3 4 ~ F}$ & NS \\
\hline Education $(\mathbf{y})$ & $13(13-17)$ & $13(13-17)$ & 0.37 \\
\hline BMI & $16.5(15.3-17.6)$ & $20.98(19.8-23.3)$ & $<0.0001$ \\
\hline BDI-II & $15(7-24)$ & $6,5(3-7)$ & $\mathbf{0 , 0 0 0 1}$ \\
\hline
\end{tabular}

Values expressed as median and interquartile ranges. $\mathrm{P}<0.05$ is considered statistically significant.

AN-R, anorexia nervosa restricting type; C controls; BMI Body Mass Index, BDI-II Beck Depression Inventory-II.

Table 2 PSQI, ESS e SF-36 scores (AN) in AN-R and Controls 

AN-R $(n=34)$
$C(n=34)$
P
Effect size (r)

Nocturnal Sleep

\begin{tabular}{|llllll|}
\hline PSQI & AN & Controls & \\
\hline & C1 Quality of sleep & $1(1-2)$ & $1(1-1)$ & $<0.001$ & 0.41 \\
\hline C2 Sleep latency & $1.5(1-3)$ & $1(1-2)$ & 0.18 & 0.18 \\
\hline C3 Sleep duration & $1(0-3)$ & $0(0-1)$ & 0.02 & 0.30 \\
\hline C4 Sleep Efficiency & $1(0-2)$ & $0(0-0)$ & $\mathbf{0 . 0 0 2}$ & 0.38 \\
\hline C5 Sleep Disorders & $2(1-2)$ & $1(1-1)$ & $\mathbf{0 . 0 0 3}$ & 0.35 \\
\hline C6 Hypnotic drug use & $0(0-0)$ & $0(0-0)$ & 0.40 & 0.07 \\
\hline C7 Diurnal function & $1(0-2)$ & $1(0-1)$ & $\mathbf{0 . 0 0 4}$ & 0.37 \\
\hline Global Score & $8(6.75-12)$ & $5(3-6.25)$ & $<0.001$ & 0.58 \\
\hline PSQI>5 & $28 / 34$ & $10 / 34$ & $\mathbf{p}<$ & j=0.71 \\
\hline
\end{tabular}

\section{Daytime Somnolence}

$\begin{array}{lllll}\text { ESS } & 7(3-10) & 7.5(5-10) & 0.7 & 0.10 \\ \text { ESS }>10 & 8 / 34 & 3 / 34 & 0.09 & j=0.28\end{array}$

Quality of life SF-36

\begin{tabular}{llllll} 
SF-36 & PF & $90(75-95)$ & $100(95-100)$ & $<0.001$ & 0.56 \\
\cline { 2 - 3 } & $50(25-100)$ & $100(100-100)$ & $<0.001$ & 0.47 \\
\hline $\mathrm{BP}$ & $73(60-86.7)$ & $74(72-84)$ & 0.33 & 0.13 \\
\hline $\mathrm{GH}$ & $57(42-67)$ & $77(67-87)$ & $<0.001$ & 0.61 \\
\hline $\mathrm{VT}$ & $50(30-60)$ & $62.5(55-70)$ & $<0.001$ & 0.47 \\
\hline $\mathrm{SF}$ & $50(37.5-62.5)$ & $75(62.5-75)$ & $<0.001$ & 0.52 \\
\hline $\mathrm{RE}$ & $33.3(0-100)$ & $100(33-100)$ & $\mathbf{0 . 0 0 1}$ & 0.43 \\
\hline $\mathrm{MH}$ & $48(28-60)$ & $72(64-84)$ & $<0.001$ & 0.72 \\
\hline $\mathrm{PCS}$ & $49,9(45,1-$ & $54,91(52.6-$ & $\mathbf{0 . 0 1}$ & 0.34 \\
\hline $\mathrm{MCS}$ & $56,3)$ & $57,3)$ & $62.7(56.4-67.1)$ & $<0.001$ & 0.65
\end{tabular}


Values expressed as median (interquartile deviation). Mann-Whitney non-parametric statistical test for unpaired samples. $p<0.05$ considered statistically significant. AN-R, anorexia nervosa restricting type; $C$ control group; PSQI Pittsburgh Sleep Quality Index, ESS Epworth Sleepiness Scale; SF-36 domain: PF Physical Operation, RP Role limitations due to physical problems; BP Bodily Pain; GH General Health; VT Vitality; SF social functioning; RE role limitations due to emotional problems; MH mental health, MCS mental component summary; PCS physical component summary.

Table 3 Comparison in PSQI, ESS, BDI and SF-36 scores between AN with sleep disorders (PSQI > 5, ANSD) and AN without sleep disorders (PSQI $\leqq 5$ ANwSD)

\begin{tabular}{|c|c|c|c|c|c|}
\hline & & ANSD $(n=28)$ & ANwSD $(n=6)$ & $\mathbf{P}$ & Effect size (r) \\
\hline PSQI & Global Score & $9(8-13)$ & $4(3-5)$ & 0.0001 & 1 \\
\hline ESS & & $7(3-10.5)$ & $6(4-8)$ & 0.96 & 0.01 \\
\hline BDI-II & & $13(8-22,5)$ & $9,5(8-12)$ & 0.35 & 0.13 \\
\hline \multicolumn{6}{|c|}{ Health-related quality of life } \\
\hline \multirow[t]{10}{*}{ SF-36 } & PF & $87.5(72.5-97.5)$ & $90(85-95)$ & 0.96 & 0.01 \\
\hline & $\mathrm{RP}$ & $50(0-100)$ & $100(50-100)$ & 0.17 & 0.34 \\
\hline & BP & $68(51.5-100)$ & $87(60-100)$ & 0.52 & 0.16 \\
\hline & $\mathrm{GH}$ & $54.5(43.5-63.5)$ & $64.5(27-72)$ & 0.45 & 0.19 \\
\hline & VT & $50(30-60)$ & $47.5(35-65)$ & 0.76 & 0.07 \\
\hline & SF & $56.25(31.2-65)$ & $50(50-50)$ & 0.66 & 0.11 \\
\hline & $\mathrm{RE}$ & $33.3(0-100)$ & $50(0-100)$ & 0.69 & 0.10 \\
\hline & $\mathrm{MH}$ & $48(28-60)$ & $44(20-60)$ & 0.92 & 0.03 \\
\hline & PCS & $49.1(45.1-55.9)$ & $55.5(49.1-58.8)$ & 0,27 & 0.28 \\
\hline & MCS & $51.6(44,5-54,7)$ & $50.61(45-56,6)$ & 0.94 & 0.01 \\
\hline
\end{tabular}

Values expressed as median (interquartile range). Non-parametric statistical test Mann-Whitney for independent samples. $P<0.05$ considered statistically significant. Effect size $(r)$ rank biserial correlation; ANSD, anorexia nervosa with sleep disorders (PSQI > 5); ANwSD anorexia nervosa in the absence of sleep disorders (PSQI $\leqq 5)$; PSQI Pittsburgh Sleep Quality Index, ESS Epworth Sleepiness Scale; SF-36 domain: PF Physical Operation, RP Role limitations due to physical problems; BP Bodily Pain; GH General Health; 
VT Vitality; SF social functioning; RE role limitations due to emotional problems; MH mental health, MCS mental component summary; PCS physical component summary.

Table 4. Correlation between night sleep quality (PSQI global score) and quality of life (SF36), BMI, education and BDI in AN-R

\begin{tabular}{|lll|}
\hline SF-36 & \multicolumn{2}{l|}{ AN-R (N=34) } \\
\hline PF & -0.03 & 0.82 \\
\hline RP & -0.35 & $0.03 *$ \\
\hline BP & -0.14 & 0.42 \\
\hline GH & -0.10 & 0.56 \\
\hline PCS & -0.21 & 0.23 \\
\hline VT & 0.01 & 0.93 \\
\hline SF & -0.07 & 0.68 \\
\hline RE & -0.03 & 0.84 \\
\hline MH & -0.12 & 0.46 \\
\hline MCS & -0.07 & 0.67 \\
\hline BMI & 0.19 & 0.26 \\
\hline Education (y) & $\mathbf{0 . 3 8}$ & $\mathbf{0 . 0 2 *}$ \\
\hline BDI-II & 0.1 & 0.57 \\
\hline
\end{tabular}

SF-36 domain: PF Physical Operation, RP Role limitations due to physical problems; BP Bodily Pain; GH General Health; VT Vitality; SF social functioning; RE role limitations due to emotional problems; $\mathrm{MH}$ mental health, MCS mental component summary; PCS physical component summary, BDI-II Beck Depression Inventory. Non parametric Spearman correlation test was performed. $\mathrm{P}<0.05$ was considered statistically significant.

\section{Figures}



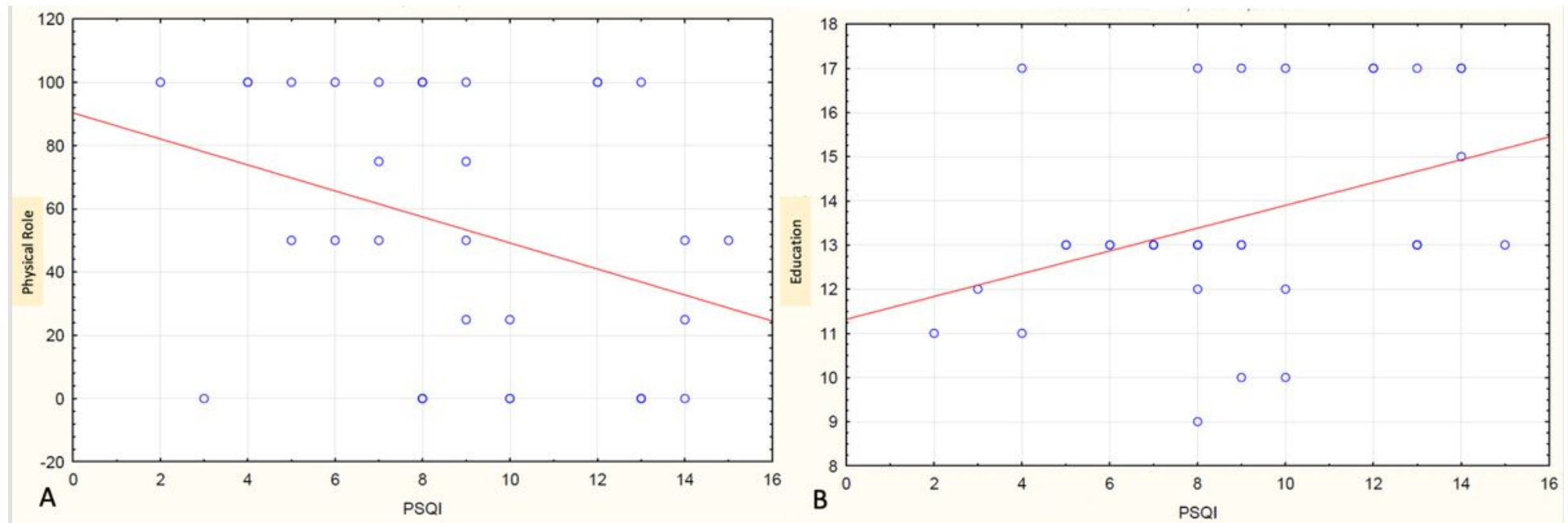

Figure 1

A Correlation between PSQI global score and Physical Role of SF36 in AN-R (Spearman nonparametric correlation test, $r=-0.35 p=0.03$ ). B Correlation between PSQI global score and Education level (years) in AN (Spearman nonparametric correlation test, $r=0.38 p=0.02$ ). 